\title{
Mitochondrial diseases: advances and issues
}

This article was published in the following Dove Press journal:

The Application of Clinical Genetics

15 February 2017

Number of times this article has been viewed

\section{Mauro Scarpelli' \\ Alice Todeschini \\ Irene Volonghi ${ }^{2}$ \\ Alessandro Padovani ${ }^{2}$ \\ Massimiliano Filosto ${ }^{2}$ \\ 'Department of Neuroscience, Unit of Neurology, Azienda Ospedaliera Universitaria Integrata Verona, Verona, Italy; ${ }^{2}$ Center for Neuromuscular Diseases and Neuropathies, Unit of Neurology, ASST "Spedali Civili", University of Brescia, Brescia, Italy}

Correspondence: Massimiliano Filosto Center for Neuromuscular Diseases and Neuropathies, Unit of Neurology, ASST Spedali Civili and University of Brescia, Piazzale Spedali Civili I, 25100 Brescia, Italy

Tel +390303995632

Fax +39030 3384086

Email massimiliano.filosto@unibs.it
Abstract: Mitochondrial diseases (MDs) are a clinically heterogeneous group of disorders caused by a dysfunction of the mitochondrial respiratory chain. They can be related to mutation of genes encoded using either nuclear DNA or mitochondrial DNA. The advent of next generation sequencing and whole exome sequencing in studying the molecular bases of MDs will bring about a revolution in the field of mitochondrial medicine, also opening the possibility of better defining pathogenic mechanisms and developing novel therapeutic approaches for these devastating disorders. The canonical rules of mitochondrial medicine remain milestones, but novel issues have been raised following the use of advanced diagnostic technologies. Rigorous validation of the novel mutations detected using deep sequencing in patients with suspected $\mathrm{MD}$, and a clear definition of the natural history, outcome measures, and biomarkers that could be usefully adopted in clinical trials, are mandatory goals for the scientific community. Today, therapy is often inadequate and mostly palliative. However, important advances have been made in treating some clinical entities, eg, mitochondrial neuro-gastrointestinal encephalomyopathy, for which approaches using allogeneic hematopoietic stem cell transplantation, orthotopic liver transplantation, and carrier erythrocyte entrapped thymidine phosphorylase enzyme therapy have recently been developed. Promising new treatment methods are being identified so that researchers, clinicians, and patients can join forces to change the history of these untreatable disorders.

Keywords: mitochondrial diseases, therapy, MNGIE

\section{Introduction}

Mitochondrial diseases (MDs) encompass a broad group of disorders that may affect children and adults and are among the most common inherited neuromuscular disorders with a minimum prevalence of around 1:5,000 live births. ${ }^{1}$ They may be manifested in a tissue-specific or a multisystem manner. ${ }^{2}$ Even though tissues with high energy demand, such as brain, muscle, and eye, are more frequently involved, patients' phenotype could be extremely variegated and heterogeneous, largely but not solely depending on the classical "rules" of mitochondrial medicine: dual genetic control (nuclear DNA [nDNA] and mitochondrial DNA [mtDNA]), level of heteroplasmy (percentage of mutated DNA in single cells and tissues), tissue energy demand, maternal inheritance, and mitotic segregation. ${ }^{2}$

MDs are usually strictly considered diseases caused by a biochemical defect of the respiratory chain $(\mathrm{RC}) .^{3}$ This definition has been progressively expanded during recent years in parallel with the growth of knowledge on the role of mitochondrial- and nuclear-derived proteins in mitochondrial functions and the role of nuclear genes in 
regulating mitochondrial homeostasis and functioning, now also including defects in the lipid milieu, in mitochondrial translation, and in mitochondrial fission and fusion. ${ }^{3,4}$

Last but not least, mitochondrial impairment plays an important role in the pathogenesis of many neurodegenerative diseases, including Alzheimer's disease, Parkinson's disease, Huntington's disease, and amyotrophic lateral sclerosis, and a growing number of studies are being developed in this field. ${ }^{5}$ Next generation sequencing/whole exome sequencing (NGS/WES) approaches will provide valuable information on the genes implicated in these disorders and could better define the impact of mitochondrial dysfunction on their pathogenesis.

A deeper knowledge of the mechanisms regulating mitochondrial function and interactions between mtDNA and nDNA in single cellular systems and different cell subtypes is very important for revealing the role of mitochondria in neurodegenerative processes, also with the aim of identifying novel and more specific therapies.

\section{Diagnosing mitochondrial diseases}

The mitochondrial genome remains of crucial importance in the pathogenesis of many MD, and it should be analyzed in every suspected MD in which there is no clear clinical and biochemical evidence of a nuclear origin of the disease. ${ }^{6}$

Due to the specific "rules" of mitochondrial medicine, if pathogenic mtDNA variants are suspected, the tissue in which mtDNA is studied is decisive in achieving a correct diagnosis. ${ }^{2}$ Although slightly invasive, muscle biopsy is the gold standard for the diagnostic workup in both children and adults because it enables us to obtain valuable morphological, biochemical, and molecular data. ${ }^{3}$ Moreover, muscle is sometimes the only tissue in which the molecular defect is present, especially in adult patients with a pure myopathic phenotype. ${ }^{7}$ On the one hand, muscle tissue could reveal the presence of single mtDNA deletions, classically found in sporadic cases of chronic progressive external ophthalmoplegia, or point mutation in transfer RNAs or in gene-encoding structural subunits of the RC complexes; on the other hand, a Southern blot analysis of mtDNA extracted from muscle tissue could show multiple deletions and/or depletion of mtDNA, referring to a mutation of the nDNA.

As alternatives to muscle, more available tissues for molecular analysis are urinary epithelium and skin fibroblasts that could also be easily used for experimental studies. $^{8}$

\section{Diagnostic and therapeutic advances}

There is currently a large availability of powerful diagnostic technologies, especially in the field of genetics. Studying thousands of patients' genomes through NGS and WES approaches is going to revolutionize the diagnostic process in mitochondrial medicine, allowing us to obtain molecular diagnosis in a growing number of previously unresolved cases and revealing novel molecular changes and previously unknown genetic and pathogenic mechanisms. ${ }^{9}$ For instance, mutations with variable penetrance, large-scale DNA rearrangements, and mutations in known genes with previously unreported "mitochondrial" phenotypes (ie, genes universally known to cause neurodegenerative disorders as $S P G 7$ mutations, usually related to spastic paraplegia, can cause chronic external ophthalmoplegia) have been identified, as well as variants in genes encoding proteins the role of which in the mitochondrial function has been unknown for a long time (ie, MPV17, which has only recently been demonstrated to serve as a nonselective channel with a pore diameter of 1.8 $\mathrm{nm}$ that contributes to mitochondrial homeostasis under different conditions). ${ }^{10-12}$

On the other hand, patients with RC enzyme deficiency suggesting MD may harbor pathogenic mutations in genes apparently not related to mitochondrial function, and therefore they ultimately could be affected by other types of disorder in which RC abnormalities may be secondary to various cellular processes, including neuromuscular transmission, calcium metabolism, and abnormal messenger RNA degradation. ${ }^{13}$

Therapies for MD are still limited, and in most cases only targeted at symptoms. Traditionally, patients have been treated with different vitamins, cofactors, and nutritional supplements. ${ }^{14}$ However, these "mitochondrial cocktails" do not have a major therapeutic impact on most MD. ${ }^{15}$

The large advances made in recent years, through the development of molecular genetic techniques for understanding the molecular bases and pathophysiological processes underlying MDs, have given a new momentum to research on effective treatments, and a big effort in developing new therapeutic approaches is offering patients hope for changing the natural history of these devastating disorders.

Although extremely promising, therapies directed toward mtDNA-related disorders are very much at the preclinical stage, and they require the adoption of rigorous clinical trials in the clinical setting. ${ }^{15}$

A promising therapeutic strategy is to force a shift in heteroplasmy, thereby reducing the ratio of mutant to wild-type 
genomes (heteroplasmic shifting), which can be obtained by selectively inhibiting the replication of mutant genomes. ${ }^{16}$ Several research groups worldwide are particularly interested in techniques aimed at preventing the transmission of maternally inherited MD. Reproductive technologies designed to uncouple the inheritance of mtDNA from nDNA may enable affected women to have a genetically related child with a greatly reduced risk of mtDNA-related disease, although they do not guarantee prevention. ${ }^{17}$

From a clinical perspective, therapies for nuclear generelated enzyme defects are at a more advanced stage. In some cases, etiological therapies are focused on restoring or bypassing specific enzyme or cofactor defects.

MD caused by defects in Coenzyme Q10 biosynthesis could be effectively treated with oral coenzyme supplementation, and riboflavin supplementation has been found to be beneficial in adults with riboflavin transporter disorders. ${ }^{18,19}$

Stem cell therapy is being used in specific contexts, and gene therapy trials have been successfully evaluated in mouse models. ${ }^{14,20}$

To date, the most important advances have been made in treating mitochondrial neuro-gastrointestinal encephalomyopathy (MNGIE), one of the rarest MDs that may benefit from available therapies, potentially improving life expectancy in the affected patients.

Certainly, MNGIE can be considered a prototype of how it may be possible to treat a MD related to a specific enzyme deficiency.

This is a devastating mitochondrial disorder caused by deficiency of the thymidine phosphorylase (TP) enzyme with consequent accumulation of nucleosides and an imbalance in the mitochondrial nucleotides pool. ${ }^{21}$ The clinical picture includes progressive gastrointestinal dysmotility, cachexia, ptosis and ophthalmoparesis, peripheral neuropathy, and diffuse leukoencephalopathy, which usually lead to death in early adulthood. ${ }^{21,22}$

Excesses of nucleosides should be cleared from MNGIE patients through a method that continuously removes or catabolizes the nucleosides, as they are also continuously produced. Attempts of this treatment strategy include: 1) enzyme replacement, through infusion of a stabilized and active form of TP protein; 2) cell replacement, either hematopoietic stem cells from adult donors and cord blood or organ transplantation; and 3) gene therapy. ${ }^{23}$

Carrier erythrocyte entrapped TP enzyme therapy has been associated with a marked reduction of plasma and urine thymidine and deoxyuridine, with the advantage that encapsulation prevents the formation of neutralizing antibodies and maintains enzyme activity for the erythrocyte life span. ${ }^{24}$ Although periodical infusions are needed, it should be considered a rescue or maintenance therapy for MNGIE patients. ${ }^{24}$

Allogeneic hematopoietic stem cell transplantation is a well-defined treatment option for this condition. ${ }^{25-27} \mathrm{~A}$ consensus conference has proposed a common approach to allogeneic hematopoietic stem cell transplantation in MNGIE based on standardization of the transplant protocol and the clinical and biochemical assessments useful in evaluating its safety and efficacy. ${ }^{27}$ This approach, however, has serious limitations including the difficulty in obtaining suitable donors, the toxicity of the conditioning regimen, and the risk of graft failure and graft-vs-host disease. In addition, MNGIE patients are generally in poor medical condition at the time of the diagnosis. ${ }^{28}$ As a consequence, this treatment is associated with high morbidity and mortality rates in this particular disorder. ${ }^{26,27}$

The use of autologous hematopoietic stem cells, genetically engineered to produce normal TYMP, would eliminate those risks, and also has the intrinsic advantage of inducing immune tolerance to the recombinant therapeutic transgene product. ${ }^{29}$ Third generation lentiviral vectors were developed to express a codon-optimized TYMP transgene using the phosphoglycerate kinase promoter to provide high TYMP levels in cell lines, and proof-of-principle has been obtained in the MNGIE mouse model. ${ }^{29}$

More recently, it has been reported that AAV2/8-mediated transfer of the human TYMP coding sequence under the control of a liver-specific promoter prevents the biochemical imbalances in a murine model of MNGIE, demonstrating that the use of AAV to direct TYMP expression in liver is feasible as a potentially safe gene therapy strategy for MNGIE. ${ }^{30}$

Organ transplantation may represent a suitable alternative option for treating patients with MNGIE. Since the liver is the main organ for protein biosynthesis and transplantation success is estimated at $90 \%$ of cases, it has been proposed as a good source of TP, and MNGIE patients may likely benefit from orthotopic liver transplantation..$^{31,32}$

MNGIE belongs to a subgroup of mtDNA depletion syndromes characterized by a reduction in the mtDNA copy number and consequent mitochondrial dysfunction in the affected tissues. These diseases are caused by mutations in specific genes that disrupt deoxy ribonucleotide metabolism, which ultimately leads to limited availability of one or more deoxy ribonucleoside triphosphates, and subsequent mtDNA depletion. ${ }^{33}$ Experience and results in treating MNGIE patients could be extended to other diseases affecting similar pathways, in particular mtDNA depletion syndromes caused 
by defective nucleotide metabolism in which supplementation of appropriate precursors and/or administration of substances that inhibit their catabolism are good candidates for treatment strategies. ${ }^{33}$

\section{Current issues}

Although our knowledge about mitochondrial medicine has greatly increased, many questions still remain that have no definite answers, and several difficulties in diagnostic and pathogenic interpretation are experienced daily by mitochondriologists all over the world, for both the mtDNA-related and nDNA-related diseases.

It is generally accepted that heteroplasmy is one of the markers of pathogenicity of an mtDNA mutation. However, homoplasmic mtDNA mutations may have been inappropriately considered as benign polymorphisms in the past, whereas it has been clearly demonstrated that they could cause both tissue-specific (ie, Leber's inherited optic neuropathy) and multisystem disorders. ${ }^{34}$

The widespread availability of complete mtDNA sequencing has revealed that mtDNA mutations are much more common in the general population than was previously thought and are found in between $0.5 \%$ and $1 \%$ of the population at $>1 \%$ heteroplasmy levels. ${ }^{35}$

These findings raised the question of the pathogenic threshold of heteroplasmy and stress the need to further elucidate the other possible factors that contribute to the pathogenesis of the disease.

Classification of MD remains difficult. Although genetic classification by $\mathrm{nDNA}$ and mtDNA mutations is obviously the most reliable way to classify these disorders, this approach raises several issues including the large number of cases in which the pathogenic variants remain to be identified, and the locus and allelic heterogeneity for which mutations in different gene loci may have the same phenotypic effect and, conversely, the same pathogenic variant may cause many different clinical phenotypes (ie, the classical m.3243A $>\mathrm{G}$ single nucleotide variant). ${ }^{3}$

Nowadays, modern NGS and WES technologies permit researchers to extensively study nuclear genome, giving them the possibility to reach the correct diagnosis in many cases that were previously unsolved. ${ }^{9}$ Yet, on the other hand, a massive parallel sequencing of the nDNA unmasks a large number of variations in different genes, and it could be hard to assign a pathogenic role to each variation in a specific context. Multiple in vitro studies on isolated mutant and wild-type proteins, mutant cell characterization, modeling in yeast or other in vivo systems, including different animal species, are necessary to validate novel mutations both in genes directly linked to mitochondrial function and in genes encoding proteins not targeted toward mitochondria and therefore with roles not obviously related to mitochondrial physiology. $^{9}$

\section{Conclusion}

The field of MD is highly dynamic and new scenarios arise every day. The improvement and the large availability of highly sensitive diagnostic technologies are increasing the number of patients with a confirmed molecular diagnosis, advancing understanding of the pathogenic mechanisms, and offering the possibility to design targeted and more specific treatments for MD. ${ }^{36-43}$

International efforts to share data will facilitate this work, enabling different groups to confirm or refute putative novel pathogenic mutations as early as possible.

It remains crucially important for clinicians and researchers to have a close relationship in order to validate the results of both diagnostic and therapeutic studies.

The discovery of gene mutations linked to MD is providing clues as to how target therapies and detailed studies of genetically homogeneous clinical cohorts are required in order to best define the natural histories and to identify reliable biomarkers and features that change over a long observation period that could be used as clinical trial endpoints.

MNGIE is a prototypical example of how translational studies can reveal scientifically rational therapies. Certainly, overall survival and restoration of TP activity are main indicators of good clinical outcome during therapy. However, it is still difficult to define whether or not stabilization rather than an improvement of clinical symptoms is satisfactory.

The future looks brighter as the revolution in the diagnostic approach could prelude the revolution in the treatment options for MD.

\section{Disclosure}

All authors have read and agreed upon the contents of the paper. The authors report no conflicts of interest in this work.

\section{References}

1. Schaefer AM, McFarland R, Blakely EL, et al. Prevalence of mitochondrial DNA disease in adults. Ann Neurol. 2008;63:35-39.

2. DiMauro S, Schon EA. Mitochondrial respiratory-chain diseases. N Engl J Med. 2003;348:2656-2668.

3. Filosto M, Mancuso M. Mitochondrial diseases: a nosological update. Acta Neurol Scand. 2007;115:211-221.

4. Vafai SB, Mootha VK. Mitochondrial disorders as windows into an ancient organelle. Nature. 2012;491:374-383.

5. Filosto M, Scarpelli M, Cotelli MS, et al. The role of mitochondria in neurodegenerative diseases. J Neurol. 2011;258:1763-1774. 
6. Cui H, Li F, Chen D, et al. Comprehensive next-generation sequence analyses of the entire mitochondrial genome reveal new insights into the molecular diagnosis of mitochondrial DNA disorders. Genet Med. 2013;15:388-394.

7. Jeppesen TD, Duno M, Risom L, et al. A novel de novo mutation of the mitochondrial tRNAlys gene mt.8340G $>$ A associated with pure myopathy. Neuromuscul Disord. 2014;24:162-166.

8. Whittaker RG, Blackwood JK, Alston CL, et al. Urine heteroplasmy is the best predictor of clinical outcome in the m.3243A $>\mathrm{G}$ mtDNA mutation. Neurology. 2009;72:568-569.

9. Legati A, Reyes A, Nasca A, et al. New genes and pathomechanisms in mitochondrial disorders unraveled by NGS technologies. Biochim Biophys Acta. 2016;1857:1326-1335.

10. Pfeffer G, Gorman GS, Griffin H, et al. Mutations in the SPG7 gene cause chronic progressive external ophthalmoplegia through disordered mitochondrial DNA maintenance. Brain. 2014;137:1323-1336.

11. Mendelsohn BA, Mehta N, Hameed B, Pekmezci M, Packman S, Ralph J. Adult-onset fatal neurohepatopathy in a woman caused by MPV17 mutation. JIMD Rep. 2014;13:37-41.

12. Antonenkov VD, Isomursu A, Mennerich D, et al. The human mitochondrial DNA depletion syndrome gene MPV17 encodes a nonselective channel that modulates membrane potential. J Biol Chem. 2015;290:13840-13861.

13. Pyle A, Nightingale HJ, Griffin H, et al. Respiratory chain deficiency in non mitochondrial disease. Neurol Genet. 2015;1:e6.

14. Scarpelli M, Todeschini A, Rinaldi F, Rota S, Padovani A, Filosto M. Strategies for treating mitochondrial disorders: an update. Mol Genet Metab. 2014;113:253-260.

15. Pfeffer G, Horvath R, Klopstock T, et al. New treatments for mitochondrial disease - no time to drop our standards. Nat Rev Neurol. 2013;9:474-481.

16. Scarpelli M, Cotelli MS, Mancuso M, et al. Current options in the treatment of mitochondrial diseases. Recent Pat CNS Drug Discov. 2010;5:203-209.

17. Hyslop LA, Blakeley P, Craven L, et al. Towards clinical application of pronuclear transfer to prevent mitochondrial DNA disease. Nature. 2016;534:383-386.

18. Montini G, Malaventura C, Salviati L. Early coenzyme Q10 supplementation in primaryoenzyme Q10 deficiency. $N$ Engl J Med. 2008;358:2849-2850.

19. Foley AR, Menezes MP, Pandraud A, et al. Treatable childhood neuronopathy caused by mutations in riboflavin transporter RFVT2. Brain. 2014;137:44-56.

20. Bottani E, Giordano C, Civiletto G, et al. AAV-mediated liver-specific MPV17 expression restores mtDNA levels and prevents diet-induced liver failure. Mol Ther. 2014;22:10-17.

21. Filosto M, Scarpelli M, Tonin P, et al. Pitfalls in diagnosing mitochondrial neurogastrointestinal encephalomyopathy. $J$ Inherit Metab Dis. 2011;34:1199-1203

22. Garone C, Tadesse S, Hirano M. Clinical and genetic spectrum of mitochondrial neurogastrointestinal encephalomyopathy. Brain. 2011;134:3326-3332.

23. Hirano M, Garone C, Quinzii CM. CoQ(10) deficiencies and MNGIE: two treatable mitochondrial disorders. Biochim Biophys Acta. 2012;1820:625-631.

24. Bax BE, Bain MD, Scarpelli M, Filosto M, Tonin P, Moran N. Clinical and biochemical improvements in a patient with MNGIE following enzyme replacement. Neurology. 2013;81:1269-1271.
25. Hirano M, Martí R, Casali C, et al. Allogeneic stem cell transplantation corrects biochemical derangements in MNGIE. Neurology. 2006; 67:1458-1460.

26. Filosto M, Scarpelli M, Tonin P, et al. Course and management of allogeneic stem cell transplantation in patients with mitochondrial neurogastrointestinal encephalomyopathy. J Neurol. 2012;259:2699-2706.

27. Halter JP, Michael W, Schüpbach M, et al. Allogeneic haematopoietic stem cell transplantation for mitochondrial neurogastrointestinal encephalomyopathy. Brain. 2015;138:2847-2858.

28. Scarpelli M, Russignan A, Zombor M, et al. Poor outcome in a mitochondrial neurogastrointestinal encephalomyopathy patient with a novel TYMP mutation: the need for early diagnosis. Case Rep Neurol. 2012;4:248-253.

29. Torres-Torronteras J, Gómez A, Eixarch H, et al. Hematopoietic gene therapy restores thymidine phosphorylase activity in a cell culture and a murine model of MNGIE. Gene Ther. 2011;18:795-806.

30. Torres-Torronteras J, Viscomi C, Cabrera-Pérez R, et al. Gene therapy using a liver-targeted AAV vector restores nucleoside and nucleotide homeostasis in a murine model of MNGIE. Mol Ther. 2014;22:901-907.

31. O'Mahony CA, Goss JA. The future of liver transplantation. Tex Heart Inst J. 2012;39:874-875.

32. De Giorgio R, Pironi L, Rinaldi R, et al. Liver transplantation for mitochondrial neurogastrointestinal encephalomyopathy. Ann Neurol. 2016;80:448-455.

33. Cámara Y, González-Vioque E, Scarpelli M, et al. Administration of deoxyribonucleosides or inhibition of their catabolism as a pharmacological approach for mitochondrial DNA depletion syndrome. Hum Mol Genet. 2014;23:2459-2467.

34. McFarland R, Clark KM, Morris AA, et al. Multiple neonatal deaths due to a homoplasmic mitochondrial DNA mutation. Nat Genet. 2002;30:145-146.

35. Elliott HR, Samuels DC, Eden JA, Relton CL, Chinnery PF. Pathogenic mitochondrial DNA mutations are common in the general population. Am J Hum Genet. 2008;83:254-260.

36. Kohrogi K, Imagawa E, Muto Y, et al. Biotin-responsive basal ganglia disease: a case diagnosed by whole exome sequencing. J Hum Genet. 2015;60:381-385.

37. Garone C, Gurgel-Giannetti J, Sanna-Cherchi S, et al. A novel SUCLA2 mutation presenting as a complex childhood movement disorder. $J$ Child Neurol. In press 2016.

38. Garone C, Donati MA, Sacchini M, et al. Mitochondrial encephalomyopathy due to a novel mutation in ACAD9. JAMA Neurol. 2013;70:1177-1179

39. Chung WK, Martin K, Jalas C, et al. Mutations in COQ4, an essential component of coenzyme Q biosynthesis, cause lethal neonatal mitochondrial encephalomyopathy. J Med Genet. 2015;52:627-635.

40. Xu B, Li X, Du M, Zhou C, Fang H, Lyu J, Yang Y. Novel mutation of ND4 gene identified by targeted next-generation sequencing in patient with Leigh syndrome. J Hum Genet. In press 2016.

41. Dai H, Zhang VW, El-Hattab AW, et al. cprFBXL4 defects are common in patients with congenital lactic acidemia and encephalomyopathic mitochondrial DNA depletion syndrome. Clin Genet. In press 2016.

42. Thompson K, Majd H, Dallabona C, et al. Recurrent de novo dominant mutations in SLC25A4 cause severe early-onset mitochondrial disease and loss of mitochondrial DNA copy number. Am J Hum Genet. 2016;99:860-876.

43. Ng YS, Alston CL, Diodato D, et al. The clinical, biochemical and genetic features associated with RMND1-related mitochondrial disease. J Med Genet. In press 2016. 


\section{Publish your work in this journal}

The Application of Clinical Genetics is an international, peer-reviewed open access journal that welcomes laboratory and clinical findings in the field of human genetics. Specific topics include: Population genetics; Functional genetics; Natural history of genetic disease; Management of genetic disease; Mechanisms of genetic disease; Counselling and ethical issues; Animal models; Pharmacogenetics; Prenatal diagnosis; Dysmorphology. The manuscript management system is completely online and includes a very quick and fair peer-review system, which is all easy to use. Visit http://www.dovepress.com/testimonials.php to read real quotes from published authors.

Submit your manuscript here: https://www.dovepress.com/the-application-of-clinical-genetics-journal 